\title{
Neuropathic Pain in Elderly Patients with Chronic Low Back Painand Effects of Pregabalin: A Preliminary Study
}

\author{
Yoshihito Sakai ${ }^{1},{\text { Kenyu } \text { Ito }^{2} \text {, Tetsuro Hida }}^{2}$, Sadayuki Ito ${ }^{1}$, Atsushi Harada ${ }^{1}$ \\ ${ }^{1}$ Department of Orthopaedic Surgery, National Center for Geriatrics and Gerontology, Obu, Japan \\ ${ }^{2}$ Department of Orthopaedic Surgery, Nagoya University School of Medicine, Nagoya, Japan
}

\begin{abstract}
Study Design: Preliminary study.
Purpose: To assess the association of neuropathic pain with chronic low back pain (LBP) and the effect of pregabalin on neuropathic pain in the elderly.

Overview of Literature: Of those with chronic LBP, 37\% were predominantly presenting with neuropathic pain in young adults. Pregabalin is effective for pain in patients with diabetic neuropathy and peripheral neuralgia. No study has reported on the effects of pregabalin for chronic LBP in elderly patients yet.

Methods: Pregabalin was administered to 32 patients (age, $\geq 65$ years) with chronic LBP for 4 weeks. Pain and activities of daily living were assessed using the Neuropathic Pain Screening Questionnaire (NePSO), the pain DETECT questionnaire, visual analog scale, the Japanese Orthopedic Association score, the short form of the McGill Pain Questionnaire and the Roland Morris Disability Questionnaire. Modic change and spinal canal stenosis were investigated using magnetic resonance imaging.

Results: Altogether, $43.3 \%$ of patients had neuropathic pain according to the NePSQ and $15.6 \%$ patients had pain according to the pain DETECT. The efficacy rate of pregabalin was 73.3\%. A significant effect was observed in patients with neuropathic pain after 4 weeks of administration.

Conclusions: Neuropathic pain was slightly less frequently associated with chronic LBP in the elderly. Pregabalin was effective in reducing pain in patients with chronic LBP accompanied with neuropathic pain. Lumbar spinal stenosis and lower limb symptoms were observed in patients with neuropathic pain. We recommend the use of pregabalin for patients after evaluating a screening score, clinical symptoms and magnetic resonance imaging studies.
\end{abstract}

Keywords: Low back pain; Elderly person; Pregabalin; Treatment outcome

\section{Introduction}

Chronic low back pain (LBP) that afflicts the elderly is the most difficult to treat. In addition, it is difficult to treat elderly patients because they easily rebuff their medical treatment. The underlying pathological mechanisms and medical management of LBP in workers, which has been the subject of countless research, remains still unclear [1]. Non-specific ailments may be diagnosed in most of these patients with LBP [2]. Woolf et al. [3] proposed that

Received May 25, 2014; Revised Jul 10, 2014; Accepted Jul 18, 2014

Corresponding author: Yoshihito Sakai

Department of Orthopaedic Surgery, National Center for Geriatrics and Gerontology,

35 Gengo, Morioka-cho, Obu, Aichi Pref., Japan

Tel: +81-562-46-2311, Fax: +81-562-48-2373, E-mail: jsakai@ncgg.go.jp 
pain symptoms and syndromes should be classified into 2 broad mechanism-based pain categories: tissue-injury pain (nociceptive) or nervous system injury pain (neuropathic). Both nociceptive and neuropathic mechanisms of pain play a role in chronic LBP $[4,5]$. Freynhagen et al. [6] clarified that $37 \%$ of 7,772 patients (age, $42-63$ years) with various forms of chronic LBP predominantly had neuropathic pain. Furthermore, includingthe possibility of having an element of neuropathic pain is reaching also to $64.7 \%$ using a simple patient-based questionnaire (pain DETECT questionnaire). Kaki et al. [4] also proposed that $54.7 \%$ of patients with chronic LBP (mean age, 46.3 years) had neuropathic pain based on the Leeds Assessment of Neuropathic Symptoms and Signs pain scale. However, to our knowledge, there is no report on the association of neuropathic pain among elderly patients with chronic LBP.

Pregabalin is worldwide used for the treatment of neuropathic pain [7]. Regardless of the primary cause it is administered as the initial treatment of choice for neuropathic pain [8]. Pregabalin functions by modulating the alpha-2-delta subunit of the voltage-gated calcium channel, thus relieving neuropathic pain [9]. Randomized, placebo-controlled clinical trials have shown the efficacy of pregabalin for pain relief in patients with diabetic neuropathy and peripheral neuralgia $[10,11]$. Reports about its effect on chronic LBP in elderly patients are scarce. This study aimed to identify the association between neuropathic disorder and chronic LBP in elderly patients and to assess the efficacy of pregabalin treatment on LBP.

\section{Materials and Methods}

In this study, 32 patients (age, $\geq 65$ years) with chronic LBP were recruited and followed up for 3 months who visited the National Center for Geriatrics and Gerontology from January to December 2012. After verifying the ineffectiveness of a non-steroidal anti-inflammatory drug (NSAID) treatment for one month, all patients were assessed using the Neuropathic Pain Screening Questionnaire (NePSQ) [12] (neuropathic pain was given a score of six or more points, if present) and the pain DETECT questionnaire [6] (neuropathic pain was given a score of thirteen or more points, if present). Moreover, a 4-week treatment with $75 \mathrm{mg}$ of pregabalin was prescribed to each patient to be taken orally before bedtime. Other medications including NSAIDs were not taken allowed during the study period. The origin of LBP was classified as motion-induced LBP and walking-induced LBP and LBP was analyzed accordingly. Motion-induced LBP was defined as LBP that appears at the time of rising from bed or sitting position and walking-induced LBP as LBP that appears while standing up or walking. Pain was assessed using the visual analog scale (VAS, 0-10), the Japanese Orthopedic Association (JOA) score, sensory pain rating index (S-PRI), and affective pain rating index (A-PRI) [13] short form of the McGill Pain Questionnaire (SF-MPQ) and Roland Morris Disability Questionnaire (RDQ) [14]. Pain was evaluated every two weeks. Radiographic evaluations included the lordosis angle, scoliosis angle, sacral inclination angle, and the lumbar range of motion. Magnetic resonance imaging (MRI) was used to determine the presence of a lumbar spinal canal stenosisand a vertebral endplate signal change (Modic change [15]) and to evaluate the area of the erector spine muscle and lumbar multifidus muscle at the level of L1/2 and L4/5. Patients with leg pain intensity greater than the LBP, acute thoracolumbar spinal fracture, diabetes, dementia and/or a history of pregabalin use were excluded. All measurements were done after obtaining the patient's informed consent and no medical payments or other benefits were given. Ethical approval was given by the ethics committee of the National Center for Geriatrics and Gerontology. The statistical analysis was performed using StatView 5.0 (ABACUS, Berkeley, CA, USA). The quality of means for continuous variables was assessed using the Mann-Whitney U-test. A one-way analysis of variance for repeated measures and Fisher's protected least significant difference as the post hoc test were used to compare dependent measurements obtained before and after pregabalin administration. A $p$-value of 0.05 or less was considered statistically significant.

\section{Results}

\section{Chronic LBP in association with neuropathic pain}

We included a total of 30 patients ( 21 male and 9 female patients) with an average age of $72.0 \pm 6.2$ years (range, 65-87 years) who were followed up after the 4 -week treatment. Somnolence and dizziness in 11 patients and weight gain in 1 patient were the reported secondary adverse effects of pregabalin. Two patients discontinued the medication because of dizziness. Neuropathic pain was 
Table1. Patients with neuropathic chronic low back pain

\begin{tabular}{|c|c|c|c|c|}
\hline \multirow[b]{2}{*}{ Characteristic } & \multicolumn{2}{|c|}{ Neuropathic Pain Screening Questionnaire } & \multicolumn{2}{|c|}{ Pain DETECT questionnaire } \\
\hline & $\begin{array}{l}\text { Neuropathic pain }(+) \\
\qquad(\mathrm{n}=14)\end{array}$ & $\begin{array}{l}\text { Neuropathic pain }(-) \\
\qquad(\mathrm{n}=18)\end{array}$ & $\begin{array}{l}\text { Neuropathic pain }(+) \\
\qquad(\mathrm{n}=5)\end{array}$ & $\begin{array}{l}\text { Neuropathic pain (-) } \\
\qquad(\mathrm{n}=27)\end{array}$ \\
\hline Age (yr) & $68.9 \pm 4.6^{*}$ & $74.3 \pm 6.9^{*}$ & $67.8 \pm 3.0^{*}$ & $72.7 \pm 6.6^{*}$ \\
\hline Gender (male:female) & $9: 5$ & $12: 6$ & $4: 1$ & $17: 10$ \\
\hline Affected periods (wk) & $56.1 \pm 39.6^{*}$ & $31.6 \pm 33.8^{*}$ & $51.2 \pm 44.1$ & $34.9 \pm 31.0$ \\
\hline VAS & $6.6 \pm 1.6^{*}$ & $5.6 \pm 1.5^{*}$ & $12.1 \pm 4.9^{* *}$ & $5.4 \pm 2.9^{* *}$ \\
\hline JOA score & $18.3 \pm 3.3$ & $18.1 \pm 3.7$ & $16.4 \pm 3.8$ & $18.5 \pm 3.4$ \\
\hline $\mathrm{RDO}$ & $10.5 \pm 5.0$ & $9.7 \pm 4.2$ & $13.8 \pm 3.6$ & $9.3 \pm 4.4$ \\
\hline \multicolumn{5}{|l|}{ SF-MPO } \\
\hline S-PRI & $7.5 \pm 3.8^{* *}$ & $3.7 \pm 1.8^{* *}$ & $8.2 \pm 4.1^{*}$ & $4.9 \pm 3.0^{*}$ \\
\hline A-PRI & $1.8 \pm 1.4^{* *}$ & $0.4 \pm 0.1^{* *}$ & $2.0 \pm 1.5$ & $0.8 \pm 0.2$ \\
\hline Lower leg symptoms (+:-) & $11: 3^{*}$ & $9: 9^{*}$ & $5: 0$ & $14: 13$ \\
\hline Lordotic angle $\left({ }^{\circ}\right)$ & $35.1 \pm 10.8$ & $36.3 \pm 10.6$ & $29.4 \pm 8.1$ & $37.0 \pm 10.6$ \\
\hline Scoliuotic angle $\left({ }^{\circ}\right)$ & $2.8 \pm 3.4$ & $7.7 \pm 7.2$ & $4.0 \pm 4.5$ & $5.7 \pm 6.6$ \\
\hline Sacral inclination $\left({ }^{\circ}\right)$ & $28.8 \pm 7.1$ & $30.5 \pm 7.4$ & $25.8 \pm 6.0$ & $30.5 \pm 7.3$ \\
\hline Lumbar ROM $\left(^{\circ}\right)$ & $55.6 \pm 18.2$ & $68.2 \pm 22.4$ & $47.2 \pm 17.8$ & $65.8 \pm 20.9$ \\
\hline CSA of lumbar multifidus $\left(\mathrm{mm}^{2}\right)$ & $1098.3 \pm 299.7$ & $1058.8 \pm 354.6$ & $1040.6 \pm 191.6$ & $1082.6 \pm 348.8$ \\
\hline CSA of erector spinae $\left(\mathrm{mm}^{2}\right)$ & $2147.8 \pm 591.0$ & $1871.2 \pm 432.7$ & $1847.0 \pm 310.6$ & $2019.1 \pm 548.3$ \\
\hline Modic change (+:-) & $3: 11^{*}$ & $10: 8^{*}$ & $2: 3$ & $13: 14$ \\
\hline Lumbar canal stenosis (+:-) & $12: 2^{*}$ & $9: 9^{*}$ & $4: 1$ & $15: 12$ \\
\hline
\end{tabular}

VAS, visual analogue scale; JOA score, Japanese Orthopaedic Association score; RDQ, Roland Morris disability questionnaire; SF-MPQ, short form of the McGill pain questionnaire; S-PRI, sensory pain rating index; A-PRI, affective pain rating index; ROM, range of motion; CSA, cross sectional area. ${ }^{*} p<0.05,{ }^{* *} p<0.01$.

identified in 13 patients (43.3\%) using the NePSQ and in 5 patients (15.6\%) using pain DETECT. As indicated by both assessment methods, the neuropathic pain was significantly reduced with age. Patients who were diagnosed to have neuropathic pain using pain DETECT $(p<0.05)$ were also diagnosed to have neuropathic pain using NePSQ. As for neuropathic pain using NePSQ, the affected period was significantly long $(p<0.05)$. VAS scores indicated a significantly high incidence of neuropathic pain according to both questionnaires (NePSQ, $p<0.05$; pain DETECT, $p<0.01)$. The S-PRI score in the SF-MPQ, which is indicative of neuropathic pain, was significantly high (NePSQ, $p<0.01$; pain DETECT, $p<0.05$ ), and this score was associated with lower extremity symptoms and neuropathic pain according to the NePSQ $(p<0.05)$. According to the JOA score and RDQ, the activities of daily living did not show a significant association with the incidence of neuropathic pain. No significant association with the incidence of neuropathic pain was also observed in lumbar lordosis angle, scoliosis angle, range of motion and sacral inclination angle. On MRI evaluations, no significant association with the incidence of neuropathic pain was observed in the lumbar multifidus and erector spine muscle area. The NePSQ indicated a significantly reduction of Modic end plate change $(p<0.05)$ and a lumbar spinal canal stenosis was statistically significant in the neuropathic pain $(p<0.05)$ (Table 1$)$.

\section{Efficacy of pregabalin on the reduction of neuropathic pain}

Five patients reported an excellent pain relief, 17 patients reported a good pain relief and 8 patients reported no effect (efficacy rate, 73.3\%) after an administration of 
pregabalin for 4 weeks. The pain relief onset occurred on an average of $10.2 \pm 5.6$ days after administration. In cases with neuropathic pain, there was no significant difference in the effectiveness rate and the onset of pain relief (Fig. 1). The VAS score was reduced with time and a significant improvement was obtained at 4 weeks $(p<0.05)$. The rates of reduction in the VAS score according to both questionnaires were statistically significant in patients with neuropathic pain $(p<0.05)$ (Fig. 2$)$. The JOA score and RDQ were not significantly different in patients with neuropathic pain (Figs. 3, 4), but both evaluations showed a significant improvement in neuropathic pain after 4 weeks, according to the S-PRI of the SF-MPQ (Fig. 5).

\section{Evaluation of pregabalin according to the pathogenesis of LBP}

The results indicated that 18 patients had a motioninduced LBP and 14 patients had a walking-induced LBP. There were no significant differences in age, sex and each pain score. There was as also no significant difference in the scores of NePSQ and pain DETECT (Table 2). Pregabalin showed a significant improvement in the walkinginduced LBP and in the VAS. There was a significant reduction of motion-induced LBP after 4 weeks of medication (Fig. 6).

\section{Discussion}

The association between neuropathic pain and LBP has been demonstrated with various screening tests and this association was found to be present in $20 \%-50 \%$ of patients $[4-6,16]$. However, these studies were mainly performed in young populations. In elderly patients, owing to the influence of the spinal degeneration component, it is predicted that the rate of nociceptive pain is high, but no studies have validated this hypothesis. In the present study, the neuropathic pain screening test scores and pain DETECT indicated that $43.3 \%$ and $15.6 \%$ of elderly patients with chronic LBP had neuropathic pain, respectively. The scores were lower than those reported in previous studies on young patients. The association between neuropathic pain and chronic LBP has been attributed to the damage to nerve tissue, which generates mechanical pressure and inflammation of the nerve root due to disc degeneration or a herniated intervertebral disc [16]. Unlike nociceptive pain, neuropathic pain may easily undergo a chronic evolution and patients find it difficult
Neuropathic pain screening questionnaire

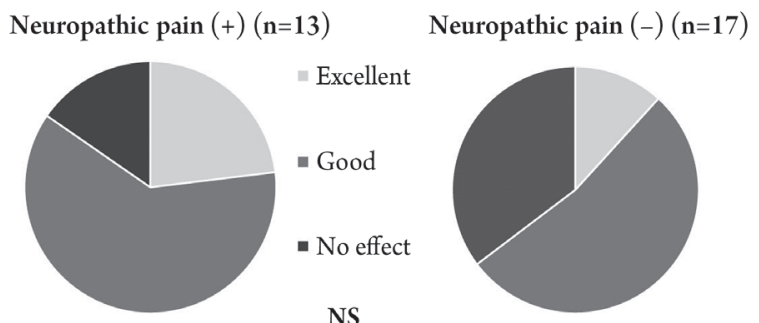

NS

Effect appearance

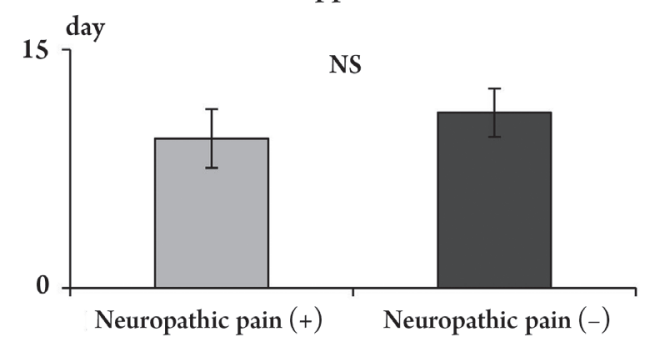

Pain DETECT questionnaire

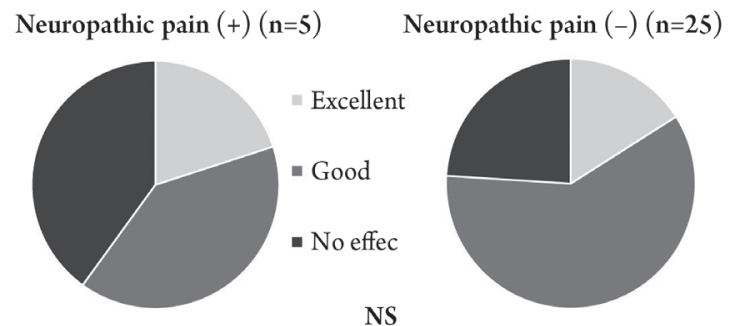

Effect appearance

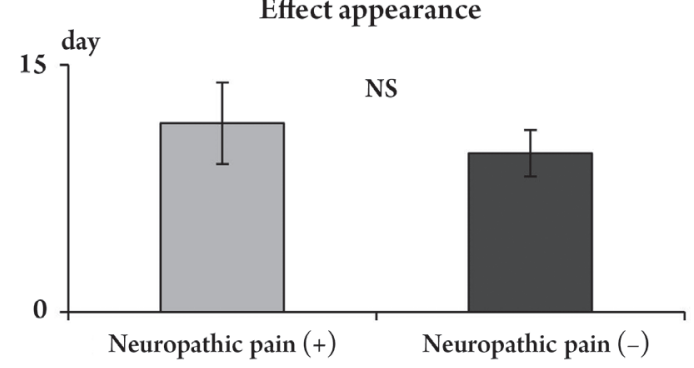

Fig. 1. Neuropathic pain and the effects of pregabalin. The evaluation of neuropathic pain by using questionnaires yielded similar results with regard to the effects of pregabalin and pain onset and did not show a significant difference between the presence and absence of neuropathic pain. NS, no significant. 


\section{Neuropathic pain screening questionnaire}

$\square$ Neuropathic pain $(+) \quad \square$ Neuropathic pain $(-)$

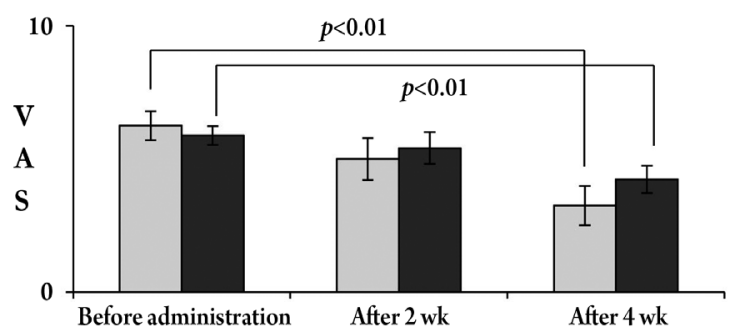

\% Improvement in VAS

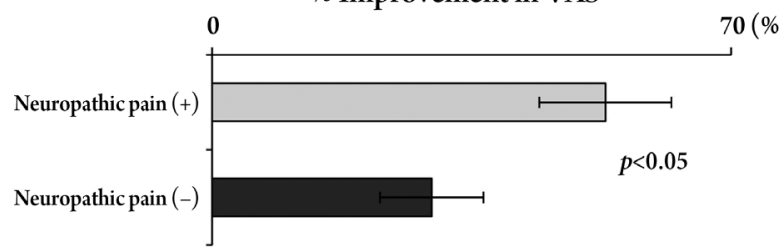

Pain DETECT questionnaire

$\square$ Neuropathic pain $(+)$
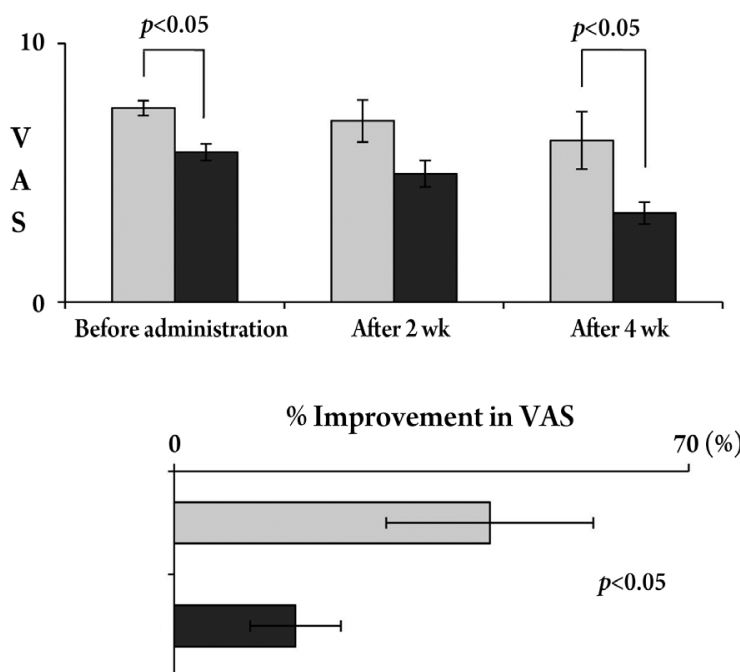

Fig. 2. Changes in visual analogue scale (VAS) scores for low back pain and the percentageof improvement according to both questionnaires. According to the Neuropathic Pain Screening Questionnaire, the VAS score for low back pain significantly improved 4 weeks after pregabalin administration, irrespective of neuropathic pain. According to the pain DETECT Questionnaire, the VAS score in patients with neuropathic pain was significantly higher than in those without neuropathic pain and this difference was seen 4 weeks after pregabalin administration. In both questionnaires, the percentage of improvement in VAS scores was significantly higher in patients with neuropathic pain than in those without.

Neuropathic pain screening questionnaire

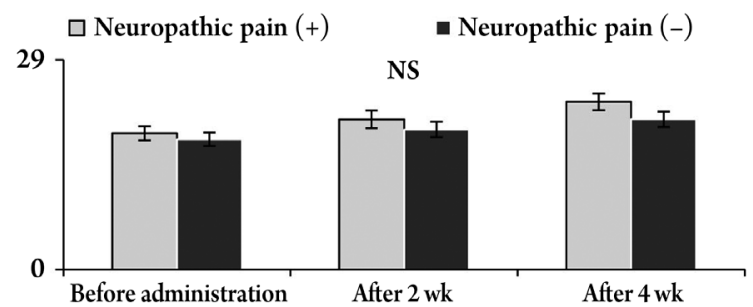

Pain DETECT questionnaire

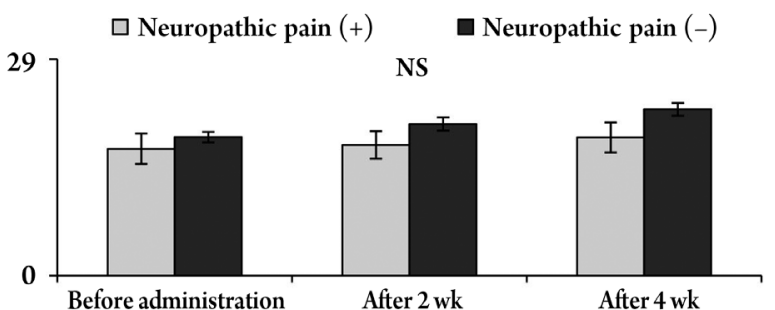

Fig. 3. Changes in the JOA score and improvement ratesaccording to both questionnaires. The JOA score improved after pregabalin administration, but there were no significant differences between neuropathic pain and nociceptive pain in both questionnaires. NS, no significant; JOA score, Japanese Orthopaedic Association score.

Neuropathic pain screening questionnaire

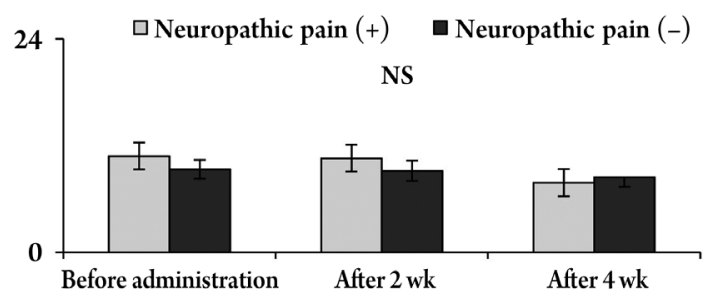

Pain DETECT questionnaire

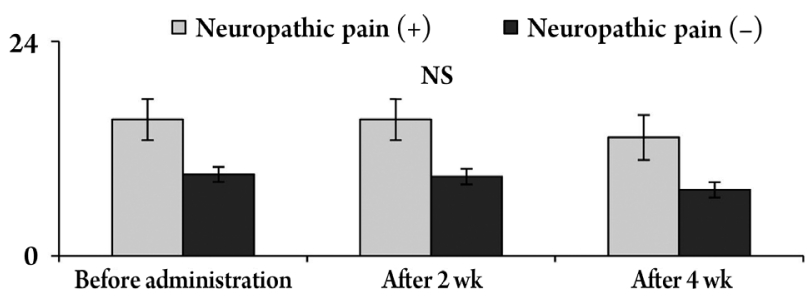

Fig. 4. Changes in the Roland Morris Disability Questionnaire (RDQ) and percent improvement according to both questionnaires. The RDO tended to improve after pregabalin administration, but there were no significant differences between neuropathic pain and nociceptive pain in both questionnaires. NS, no significant. 
Neuropathic pain screening questionnaire

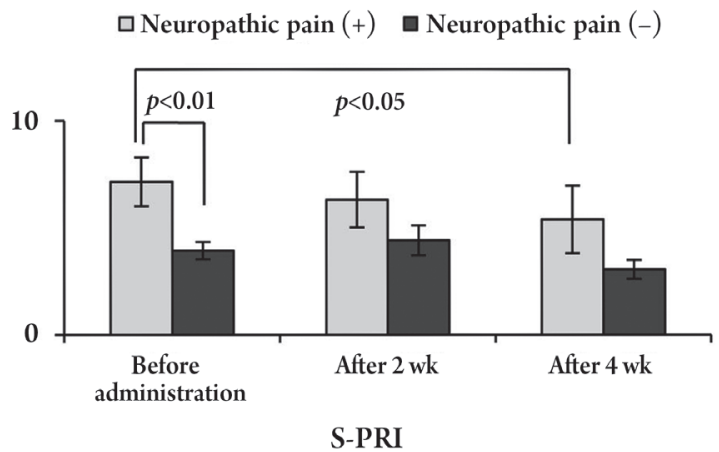

$\square$ Neuropathic pain (+) $\quad$ Neuropathic pain (-)

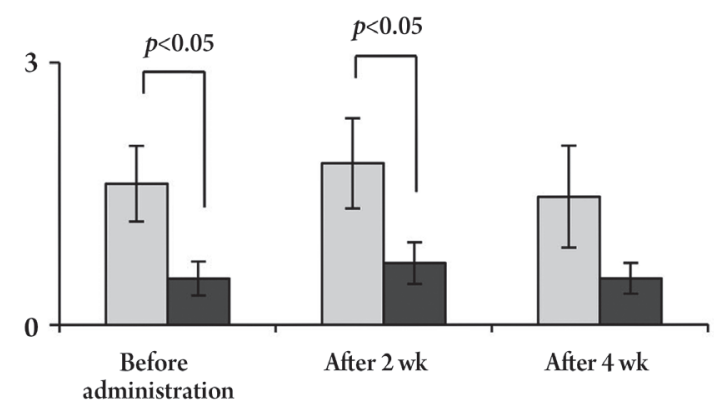

A-PRI
Pain DETECT questionnaire

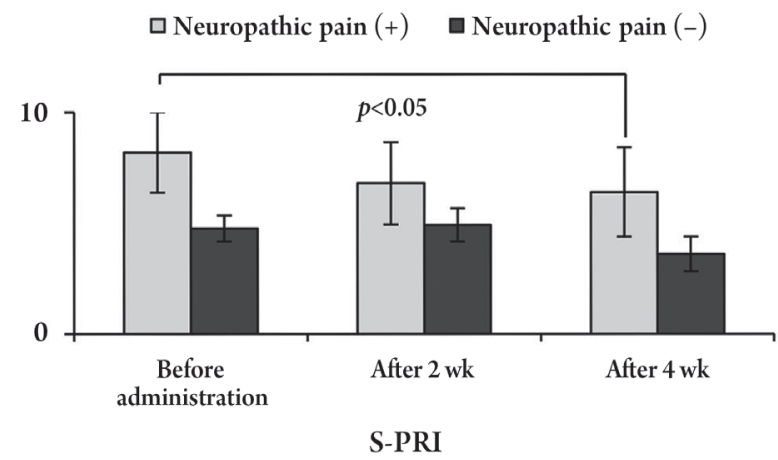

$\square$ Neuropathic pain (+) $\quad$ Neuropathic pain (-)

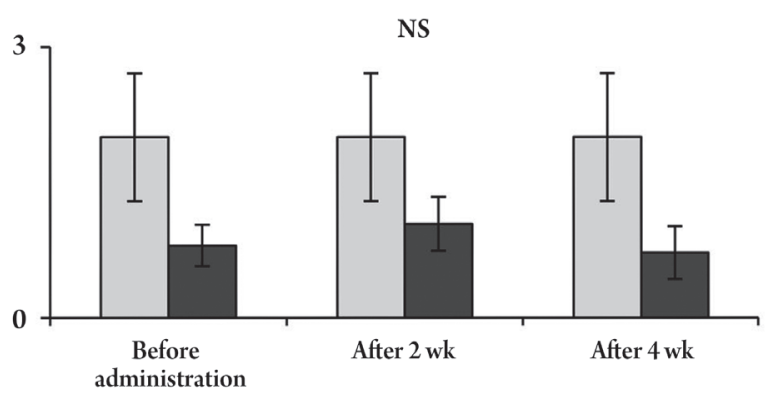

A-PRI

Fig. 5. Changes in the short form of the McGill Pain Questionnaire (SF-MPQ), including S-PRI and A-PRI, according to both questionnaires. Both S-PRI and A-PRI in the SF-MPO were significantly higher and the effect of pregabalin was greater in patients with neuropathic pain than in those without neuropathic pain. S-PRI, sensory pain rating index; A-PRI, affective pain rating index; NS, no significant.

Table 2. Evaluation of neuropathic pain according to the onset of LBP

\begin{tabular}{lcc} 
Characteristic & Motion-induced LBP $(\mathrm{n}=18)$ & Walking-induced LBP $(\mathrm{n}=14)$ \\
Age (yr) & $72.0 \pm 6.3$ & $71.8 \pm 6.7$ \\
Gender (male:female) & $14: 4$ & $7: 7$ \\
Affected periods (wk) & $40.0 \pm 34.5$ & $33.5 \pm 32.3$ \\
VAS & $6.4 \pm 1.8$ & $5.6 \pm 1.3$ \\
JOA score & $18.2 \pm 3.9$ & $18.2 \pm 3.0$ \\
RDO & $10.1 \pm 4.7$ & $9.9 \pm 4.5$ \\
SF-MPO & & $4.3 \pm 1.8$ \\
\hline S-PRI & $6.3 \pm 4.0$ & $0.7 \pm 0.3$ \\
\hline A-PRI & $1.2 \pm 0.3$ & $4.0 \pm 0.8$ \\
Neuropathic Pain Screening Questionnaire & $5.0 \pm 0.7$ & $7.3 \pm 0.9$ \\
\hline Pain DETECT Questionnaire & $9.2 \pm 1.4$ & \\
\hline
\end{tabular}

LBP, low back pain; VAS, visual analogue scale; JOA score, Japanese Orthopaedic Association score; RDQ, Roland Morris disability questionnaire; SF-MPQ, short form of the McGill Pain Questionnaire; S-PRI, sensory pain rating index; A-PRI, affective pain rating index.

to adapt to such chronicity [17]. Furthermore, if the nociceptive pain presents repeatedly, a peripheral or central sensitization may occur. If this pain becomes chronic, it is said that both nociceptive and neuropathic pain compo- 


\section{Effect of pregabalin}

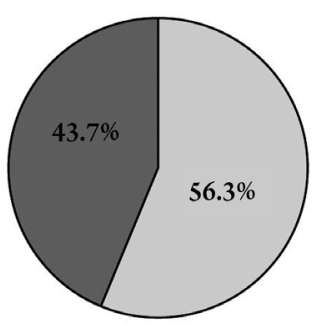

Motion-induced LBP

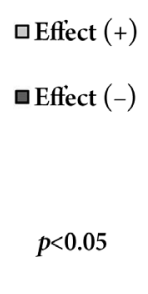

Walking-induced LBP

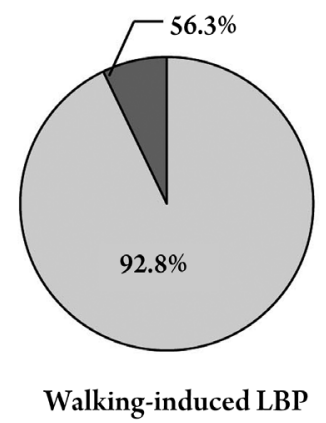

The time course of VAS

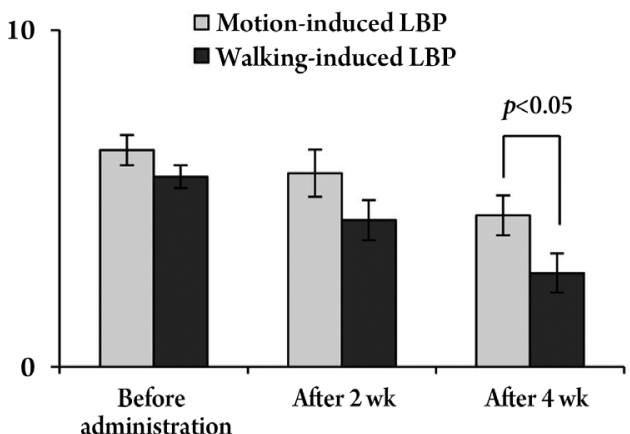

Fig. 6. Effect of pregabalin on motion- and walking-induced low back pain (LBP). Pregabalin was significantly more effective in patients with walking-induced LBP than in those with motion-induced LBP. The VAS score significantly reduced after 4 weeks of pregabalin administration. VAS, visual analogue scale.

nents are present [18]. Although the elderly have a high possibility of suffering from joint deformity, inflammation and instability that may cause nociceptive pain, the rate of neuropathic pain may increase depending on the chronicity or duration of neuropathic pain, as indicated by our study.

The differential diagnosis for nociceptive pain and neuropathic pain is explained by the diagnostic flow chart [19] for neuropathic pain of the International Association for the Study of Pain. The definite diagnosis of neuropathic pain is established by the presence of disturbance of sensation in a nerve-controlled domain and nerve damage or by the confirmed diagnosis indicative of neuropathic pain. However, its generally not easy to make the differential diagnosis in a clinical setting. Furthermore, in LBP, owing to the involvement of both nociceptive and neuropathic pain, it seems impossible to diagnose neuropathic pain using this flow chart. In fact, various diagnostic tools are used in clinical practice to rule out the possibility of neuropathic pain more easily. Of these, we compared the neuropathic pain screening tool developed specifically for the Japanese with the pain DETECT that is used globally. Both screening tools contain similar questions, however, pain DETECT has more items compared with NePSQ which is oriented as a simplified questionnaire. Our results suggest that pain DETECT is more stringent than the neuropathic pain screening tool and it is thought that pain DETECT is more suitable in order to diagnose neuropathic pain with certainty. However, since LBP involves a mixture of pain types, the direction given by the NePSQ is helpful in determining a treatment course. Moreover, we evaluated neuropathic pain using the SF-MPQ as well so that emotional and subjective explanations could also be considered.

The possibility of a nervous involvement in LBP has been considered because of reports of reduced LBP after a nerve root block [20,21], spinal pain mapping [22] and decompressive surgery $[23,24]$. Because intermittent claudication, a main condition of a lumbar spinal canal stenosis causes pain in the extremities after maintaining an upright standing position and/or walking, it is suspected that LBP exacerbated during walking involves a similar neuropathy. Takahashi et al. [25], using surface electromyography, reported that an exacerbation of LBP while walking occurred due to the dysfunction of back muscles, characterized by an increased pressure and fatigue of the muscles. The compression of the cauda equine and/or lumbar nerve roots such as that noted in a lumbar spinal canal stenosis results in a multifidus muscle impairment; this impairment possibly causes neuropathy because the lumbar multifidus muscle that receives the medial branch of the lumbar dorsal rami has a segmental nerve supply [26]. We compared the effect of pregabalin for walking- and movement-induced LBP, which mainly occur while standing. Thus, the MRI findings of patients with LBP considered to have neuropathic pain according to a screening test indicate a degeneration of the end plate; however, there were many cases of spinal canal stenosis. Although our study does not confirm the existence of neuropathic LBP, we consider that our findings support the hypothesis that a treatment with pregabalin has a greater effect in patients with neuropathic pain as determined using screening tools and patients with walkinginduced LBP, leading to the feasibility of pregabalin for 
the treatment of LBP.

There is a report on the efficacy of pregabalin in the treatment of lumbar radiculopathy [27] and another on its validity for the perioperative administration in lumbar surgeries [28] in an orthopedic setting. However, there are no reports on the validity of pregabalin on chronic LBP, except for a report by Romano et al. [29] in which a combination of pregabalin with a selective cyclooxygenase-2 inhibitor was used. This combination therapy showed better results than a pregabalin monotherapy in patients with chronic LBP aged 75 years at maximum (mean age, 53 years). In the present study, we found that the validity of pregabalin was accepted, irrespective of the existence of neuropathic pain in elderly patients with chronic LBP in this research. Nociceptive pain shifts to neuropathic pain once it becomes chronic, but this cannot be completely perceived by a screening score. However, the level of pain relief was greater in patients with neuropathic pain. The effects of pregabalin were notably seen in the VAS or SF-MPQ that may reflect the neuropathic pain, whereas no improvement was seen in the JOA score and RDQ that represent the evaluation in activities of daily living. This seems to be attributed to the mechanism of the pregabalin effect. Pregabalin is known for its anticonvulsant functions by modulating the alpha-2-delta subunit of the voltage-gated calcium channel mainly for relieving the neuropathic pain [9], whereas opioids affecting affect the central nervous system have their effects in the improvement of activities of daily living in patients with chronic LBP [30]. While determining the effects of pregabalin on chronic LBP, we should consider its clinical incidence, a screening score or VAS and the neuropathic pain using the SF-MPQ and the existence of LBP secondary to a pathology such as cauda equina neuropathy, nervous system conditions affecting lower limbs, spinal canal stenosis and walking-induced LBP.

The primary limitation of the analyses is that those were not powered to assess the effect of pregabalin. Thus, we take the results as preliminary and in need of replication. This study presented with a short follow-up period of 4 weeks, therefore long-term outcomes may be different from our data. To our knowledge, there are no previous reports focusing on neuropathic LBP in the elderly patients and the effects of pregabalin. For further investigation, it is required to assess the diagnosis of neuropathic LBP and a reasonable therapeutic administration.

\section{Conclusions}

Our study results suggest that pain DETECT is more suitable than the neuropathic pain screening tool in order to diagnose neuropathic pain of chronic LBP. However, the direction given by the NePSQ is helpful in determining a treatment course because LBP involves a mixture of pain types. Since neuropathic pain is considered as a part of the underlying mechanisms of a chronic LBP, pregabalin could also be adopted for the elderly patients with chronic LBP and with neuropathic pain.

\section{Conflict of Interest}

No potential conflict of interest relevant to this article was reported.

\section{References}

1. Rudy TE, Weiner DK, Lieber SJ, Slaboda J, Boston JR. The impact of chronic low back pain on older adults: a comparative study of patients and controls. Pain 2007;131:293-301.

2. Abraham I, Killackey-Jones B. Lack of evidencebased research for idiopathic low back pain: the importance of a specific diagnosis. Arch Intern Med 2002;162:1442-4.

3. Woolf CJ, Bennett GJ, Doherty M, et al. Towards a mechanism-based classification of pain? Pain 1998; 77:227-9.

4. Kaki AM, El-Yaski AZ, Youseif E. Identifying neuropathic pain among patients with chronic low-back pain: use of the Leeds Assessment of Neuropathic Symptoms and Signs pain scale. Reg Anesth Pain Med 2005;30:422-8.

5. Freynhagen R, Baron R, Tolle T, et al. Screening of neuropathic pain components in patients with chronic back pain associated with nerve root compression: a prospective observational pilot study (MIPORT). Curr Med Res Opin 2006;22:529-37.

6. Freynhagen R, Baron R, Gockel U, Tolle TR. painDETECT: a new screening questionnaire to identify neuropathic components in patients with back pain. Curr Med Res Opin 2006;22:1911-20.

7. Ogawa S, Satoh J, Arakawa A, Yoshiyama T, Suzuki M. Pregabalin treatment for peripheral neuropathic pain: a review of safety data from randomized con- 
trolled trials conducted in Japan and in the west. Drug Saf 2012;35:793-806.

8. Moulin DE, Clark AJ, Gilron I, et al. Pharmacological management of chronic neuropathic pain-consensus statement and guidelines from the Canadian Pain Society. Pain Res Manag 2007;12:13-21.

9. Field MJ, Li Z, Schwarz JB. Ca2+ channel alpha2delta ligands for the treatment of neuropathic pain. J Med Chem 2007;50:2569-75.

10. Vranken JH, Dijkgraaf MG, Kruis MR, van der Vegt MH, Hollmann MW, Heesen M. Pregabalin in patients with central neuropathic pain: a randomized, double-blind, placebo-controlled trial of a flexibledose regimen. Pain 2008;136:150-7.

11. Freynhagen R, Grond S, Schupfer G, et al. Efficacy and safety of pregabalin in treatment refractory patients with various neuropathic pain entities in clinical routine. Int J Clin Pract 2007;61:1989-96.

12. Ogawa $S$. Development of new screening questionnaire to identify neuropathic components in Japanese patients with chronic pain. Pain Clinic 2010;31:118794.

13. Melzack R. The McGill Pain Questionnaire: major properties and scoring methods. Pain 1975;1:277-99.

14. Roland M, Morris R. A study of the natural history of back pain. Part I: development of a reliable and sensitive measure of disability in low-back pain. Spine (Phila Pa 1976) 1983;8:141-4.

15. Modic MT, Steinberg PM, Ross JS, Masaryk TJ, Carter JR. Degenerative disk disease: assessment of changes in vertebral body marrow with MR imaging. Radiology 1988;166:193-9.

16. Freynhagen R, Baron R. The evaluation of neuropathic components in low back pain. Curr Pain Headache Rep 2009;13:185-90.

17. Woolf CJ; American College of Physicians; American Physiological Society. Pain: moving from symptom control toward mechanism-specific pharmacologic management. Ann Intern Med 2004;140:441-51.

18. Arendt-Nielsen L, Graven-Nielsen T. Central sensitization in fibromyalgia and other musculoskeletal disorders. Curr Pain Headache Rep 2003;7:355-61.

19. Treede RD, Jensen TS, Campbell JN, et al. Neuropathic pain: redefinition and a grading system for clinical and research purposes. Neurology 2008;70: 1630-5.

20. Gajraj NM. Selective nerve root blocks for low back pain and radiculopathy. Reg Anesth Pain Med 2004; 29:243-56

21. Murata Y, Kato Y, Miyamoto K, Takahashi K. Clinical study of low back pain and radicular pain pathways by using 12 spinal nerve root infiltration: a randomized, controlled, clinical trial. Spine (Phila Pa 1976) 2009;34:2008-13.

22. Pang WW, Mok MS, Lin ML, Chang DP, Hwang MH. Application of spinal pain mapping in the diagnosis of low back pain--analysis of 104 cases. Acta Anaesthesiol Sin 1998;36:71-4.

23. Toyone T, Tanaka T, Kato D, Kaneyama R. Low-back pain following surgery for lumbar disc herniation. A prospective study. J Bone Joint Surg Am 2004;86:8936.

24. Jolles BM, Porchet F, Theumann N. Surgical treatment of lumbar spinal stenosis. Five-year follow-up. J Bone Joint Surg Br 2001;83:949-53.

25. Takahashi I, Kikuchi S, Sato K, Iwabuchi M. Effects of the mechanical load on forward bending motion of the trunk: comparison between patients with motion-induced intermittent low back pain and healthy subjects. Spine (Phila Pa 1976) 2007;32:E73-8.

26. Bogduk N, Wilson AS, Tynan W. The human lumbar dorsal rami. J Anat 1982;134:383-97.

27. Saldana MT, Navarro A, Perez C, Masramon X, Rejas J. Patient-reported-outcomes in subjects with painful lumbar or cervical radiculopathy treated with pregabalin: evidence from medical practice in primary care settings. Rheumatol Int 2010;30:1005-15.

28. Burke SM, Shorten GD. Perioperative pregabalin improves pain and functional outcomes 3 months after lumbar discectomy. Anesth Analg 2010;110:1180-5.

29. Romano CL, Romano D, Bonora C, Mineo G. Pregabalin, celecoxib, and their combination for treatment of chronic low-back pain. J Orthop Traumatol 2009; 10:185-91.

30. Schofferman J, Mazanec D. Evidence-informed management of chronic low back pain with opioid analgesics. Spine J 2008;8:185-94. 